\title{
Safety of Dapagliflozin in Patients with Type 2 Diabetes Mellitus in Saudi Arabia: A Post Authorization Safety Study
}

\author{
Abdullah M. Alguwaihes
}

Received: April 18, 2021 / Accepted: May 28, 2021 / Published online: June 12, 2021

(C) The Author(s) 2021

\section{ABSTRACT}

Introduction: Sodium-glucose co-transporter-2 (SGLT2) inhibitors moderately reduce glucose levels in type-2 diabetes mellitus (T2DM). Some cases of diabetic ketoacidosis (DKA) were reported with SGLT2 inhibitors. However, data on the long-term safety of dapagliflozin in Saudi Arabia are lacking. The present study aimed to monitor the safety of dapagliflozin in patients with T2DM and evaluate the change in HbA1c during the observation period compared to baseline.

Methods: This was a local, prospective, singlearm, non-interventional, regulatory post-marketing study. The study was conducted in Saudi Arabia from May 2017 to September 2019. Patients received either 5 or $10 \mathrm{mg}$ dapagliflozin in conjunction with diet and exercise modifications to treat T2DM. The occurrence of adverse events was observed over the treatment duration and for 3 days after administering the last dose of dapagliflozin.

Supplementary Information The online version contains supplementary material available at https:// doi.org/10.1007/s13300-021-01092-0.

A. M. Alguwaihes $(\bowtie)$

Department of Internal Medicine, College of

Medicine, King Saud University, Riyadh,

Saudi Arabia

e-mail: aalguwaihes@ksu.edu.sa
Results: A total of 527 patients were recruited in the study, 524 of which were eligible for the statistical analysis. About $62 \%$ were males. The median (IQR) age was 52.3 (14.8) years, while the median (IQR) body mass index was 31.6 (7.7) $\mathrm{kg} / \mathrm{m}^{2}$. The median (IQR) duration since the patients were first diagnosed with T2DM was 8.78 (6.73) years. The most common comorbidities were hyperlipidemia $(51.1 \%)$ and hypertension (41.1\%). Almost three-quarters of the study population $(73.7 \%)$ received other antidiabetic medications in addition to dapagliflozin. Over a period of 12 months, a total of 106 adverse events were experienced by 65 $(12.33 \%)$ patients. Vulvovaginal pruritus $(3.1 \%)$, dysuria $(2.7 \%)$, polyuria $(1.3 \%)$, urinary tract infections $(1 \%)$, fatigue $(0.8 \%)$, and hypoglycemia $(0.8 \%)$ were among the reported adverse events. One case of DKA $(0.2 \%)$ was reported. The mean (SD) HbA1c\% level significantly decreased from 8.6 (1.6) \% at baseline to reach $7.2(1.2) \%$ after 12 months of treatment $(p<0.0001)$.

Conclusion: Dapagliflozin was found to be a well tolerated and effective treatment option for T2DM patients in Saudi Arabia. Vulvovaginal pruritus and dysuria were the most common adverse events.

Keywords: Dapagliflozin; Diabetic ketoacidosis; HbA1c; Post-authorization safety study; Saudi Arabia; SGLT2 inhibitor; T2DM; Urinary tract infection 


\section{Key Summary Points}

Why carry out this study?

Data on the long-term safety of dapagliflozin in Saudi Arabia are lacking.

Primarily, we aimed to explore the safety profile of dapagliflozin in type- 2 diabetes mellitus (T2DM) patients in Saudi Arabia and secondarily to evaluate the change in HbA1c during the 1-year observation period.

\section{What was learned from the study?}

Results of the current study demonstrate that dapagliflozin is well tolerated and effective with significantly improved HbA1c levels after 1 year of treatment. Hence, it can be incorporated into the routine clinical management of T2DM in Saudi Arabia.

Future experimental studies with larger samples are required to assess the occurrence of rare adverse events that may result from using dapagliflozin.

\section{DIGITAL FEATURES}

This article is published with digital features, including a summary slide, to facilitate understanding of the article. To view digital features for this article go to https://doi.org/10.6084/ m9.figshare.14697165.

\section{INTRODUCTION}

Diabetes mellitus disease is characterized by abnormal metabolism of carbohydrates, lipids, and proteins. It is characterized by chronic hyperglycemia due to a relative or absolute impairment of insulin secretion in addition to peripheral resistance to insulin action. In 2019, diabetes affected $>463$ million adults globally, and its prevalence is expected to increase to $>$
640 million adults by $2040[1,2]$. Type 2 diabetes mellitus (T2DM) is the most common type of diabetes in adults, amounting to approximately $90 \%$ of the cases [3-5]. Patients with diabetes are at higher risk of morbidity (e.g., cardiovascular and kidney events) and mortality than the general population [6-10].

There are different non-pharmacological strategies for glycemic control in T2DM patients; these are intensive lifestyle modification, exercise, and bariatric surgery [11-14]. However, the vast majority of patients require chronic treatment to reach and maintain their target glycemic level. Current medications for T2DM improve insulin sensitivity, delay the absorption of carbohydrates from the gastrointestinal tract, increase insulin secretion, or increase urinary glucose excretion as sodiumglucose co-transporter 2 (SGLT2) inhibitors.

Glucose is typically filtered in the kidney, where almost $90 \%$ of the filtered amount is reabsorbed in the proximal tubule by SGLT2. Thus, SGLT2 inhibitors lower blood glucose levels by promoting renal glucose excretion. Several studies demonstrated promising results for SGLT-2 inhibitors in reducing the risk of heart failure (HF) and hospitalization [15-17]. Other investigations showed improved renal outcome [17-19]. Furthermore, recent international guidelines for diabetes management have recently endorsed the use of SGLT2 inhibitors for patients with HF or chronic kidney disease (CKD) independent of HbA1c baseline or target [20-22].

Dapagliflozin is one of the selective SGLT2 inhibitors that block glucose resorption in the proximal tubule and promotes glucosuria in patients with T2DM. Additionally, dapagliflozin decreases the cardiac preload and afterload by reducing sodium reabsorption and increases sodium delivery to the distal tubule, which may downregulate sympathetic activity [23].

Dapagliflozin is indicated as monotherapy for the treatment of insufficiently controlled T2DM in adults as an adjunct to diet and exercise when metformin is considered inappropriate because of intolerance or in addition to other medicinal products for the treatment of T2DM [24]. Dapagliflozin is particularly useful for patients with $\mathrm{HF}$ or $\mathrm{CKD}$, given the 
cardiovascular and renal benefits associated with the medication, regardless of the diagnosis of diabetes $[17,18,21,23,25,26]$. Owing to the SGLT2 inhibitors' mechanism of action, they do not usually cause hypoglycemia if not combined with medications that otherwise cause hypoglycemia [27].

In clinical trials, SGLT2 inhibitors, including dapagliflozin, were associated with an increased risk of diabetic ketoacidosis (DKA) compared to dipeptidyl-peptidase IV inhibitor (DPP-4 inhibitors) [28, 29]. Moreover, patients taking SGLT2 inhibitors had an approximately two- to four-fold increased risk of vulvovaginal candidiasis in up to $15 \%$ of diabetic women $[30,31]$.

Accordingly, this post-authorization safety study (PASS) was conducted based upon a request from the local regulatory authority (Saudi Food and Drug Administration) to monitor the safety of dapagliflozin in patients with T2DM and evaluate the magnitude of HbA1c change during the observation period compared to baseline.

\section{METHODS}

\section{Study Design and Population}

This was a local, prospective, single-arm, noninterventional, regulatory, post-marketing study to monitor the safety of dapagliflozin in patients with T2DM and evaluate the HbA1c change during the observation period in realworld clinical practice. The study was conducted in three public and four private primary hospitals in Saudi Arabia between May 15, 2017, and September 15, 2019.

All participants aged $18-75$ years with T2DM who were initiating treatment with dapagliflozin, as indicated by the SFDA, were considered eligible for inclusion in the study. Participants were excluded from the study if they had previously experienced an episode of DKA as per physicians reporting, had a contraindication to dapagliflozin, or were on SGLT2 inhibitors other than dapagliflozin.

The baseline characteristics of our population, who had available baseline data during the period between May 2017 and June 2018 were previously published in early 2020 [32].

\section{Outcomes}

The primary endpoint was to describe the adverse events reported during the study whether they were serious or non-serious. These included: count and percentage of adverse events, seriousness, seriousness criteria, intensity, causality, and outcome. The secondary endpoint was to report the change in HbA1c during the observation period compared to baseline.

\section{Procedures}

Patients were treated as part of the routine practice at Saudi healthcare centers by accredited physicians. They received either $10 \mathrm{mg}$ or $5 \mathrm{mg}$ of dapagliflozin in conjunction with lifestyle modifications (diet and exercise) for the treatment of T2DM according to the label approved by the SFDA.

Patients were followed for 52 weeks, including the holy month of Ramadan. Baseline characteristics and HbA1c changes were captured at 12,24, and 52 weeks after baseline (plus or minus 2 weeks) or according to the site's standard of care. Also, a phone call assessment was conducted after the first week of Ramadan to ask patients if they have experienced any diabetic complications or adverse events and if there were any changes in comorbidities or concomitant medications.

All patients were evaluated for safety during the treatment period and for 3 days after administering the last dose to confirm the safety of the medication under routine practice or to report any unexpected adverse reactions.

Given the observational nature of the study, patients were allowed to continue taking their daily medications during their participation in the study. Discontinuation of treatment was determined by the patient's willingness to discontinue treatment and the investigator's discretion. 


\section{Data Collected}

Data items collected in the study included the following:

- Demographic characteristics including age, gender, race, height, weight, body mass index (BMI), waist circumference, heart rate, and blood pressure

- Age at T2DM diagnosis, duration of having the disease, and prior treatment of T2DM.

- Presence of diabetes complications

- Medical history (e.g., diabetes mellitus, dyslipidemia, hypertension, stroke, cardiovascular disease, hypoglycemia, etc.)

- Comorbidities, concomitant medications, history of abnormal renal or hepatic function, clinically significant laboratory data

- Duration of treatment and dapagliflozin dose used for each patient

- The number of patients experiencing serious and non-serious adverse events related to dapagliflozin. The reporting of unexpected adverse events included their severity, causality, and outcome

- HbA1c at baseline and follow-up visits

- Lipid profile [low-density lipoprotein (LDL), high-density lipoprotein (HDL), triglycerides, and cholesterol] at baseline and follow-up visits.

\section{Statistical Methodology}

During the first year post-authorization, the number of T2DM patients exposed to dapagliflozin was estimated to be 15,000 patients. According to the SFDA requirements, the study was initially planned to include 1500 patients, representing $10 \%$ of the total number of patients subjected to the medication during the first year. However, the sample size was reduced to 500 patients as dapagliflozin was not included in all hospitals' formularies.

Data were statistically described in terms of frequencies (number of cases) and valid percentages for categorical variables. Mean \pm standard deviation (SD), as well as the median and interquartile range (IQR), were used for numerical variables, and 95\% confidence interval (CI) for the mean HbA1c change from baseline was calculated. Wilcoxon signed-rank test was used to compare HbA1c levels at baseline and at follow-up visits. $p$ values $<0.05$ were considered statistically significant. All statistical calculations were done using IBM SPSS (Statistical Package for the Social Science; I.B.M. Corp, Armonk, NY, USA), release 21, for Microsoft Windows.

\section{Ethical Considerations}

This study was approved by the appropriate institutional ethics committees of all study sites, namely: Dallah Hospital (reference number: 17/0979/IRB), International Medical Center (reference number: 2017-11-081), King Abdulaziz University Medical Center (reference number: 159-18), King Khalid University Hospital (reference number: 17/0253/IRB), Specialized Medical Center Hospital (reference number: SMCIRB0004-2017), Sulaiman Al Habeeb Hospital (reference number: 17/0498/ IRB), and Tayba University Hospital (reference number: 17/0253/IRB). See table SD1 in the Electronic Supplementary Material for details.

The study was conducted in accordance with the ethical principles of the Declaration of Helsinki and Good Pharmacoepidemiology Practice. The study also adhered to the laws, regulations, and any relevant guidelines of Saudi Arabia.

Before conducting any research-related activities, all patients provided personally signed and dated informed consent documents, indicating that the patient or their legally acceptable representative was informed of all pertinent aspects of the study and the patients consented to participate in this study.

Consent for publication: all patients consented that their data might be shared after their anonymization for the purposes of scientific and medical research (e.g., with researchers), to allow public access to study information for sharing results with patients who participate in study or in publications. 


\section{RESULTS}

\section{Study Population}

A total of 527 patients were enrolled in this study. Three patients were excluded: two were aged $>75$ years old, while the third was excluded because of protocol deviation. Consequently, 524 patients who met the inclusion and exclusion criteria from seven sites were included in the statistical analysis. Of these patients, $61.5 \%$ were males. The majority (90.6\%) were Arab, while $7.1 \%$ were Asians. Median (IQR) age was 52.3 (14.8) years. Median (IQR) BMI was $31.6(7.7) \mathrm{kg} / \mathrm{m}^{2}$.

The median (IQR) duration since the patients were first diagnosed with T2DM was 8.78 (6.73) years. The most common comorbidities were hyperlipidemia (51.1\%) and hypertension $(41.1 \%)$. Less than $2 \%$ of the patients had a history of UTI over the last 12 months prior to enrollment in the study. Demographics and other baseline characteristics are presented in Table 1.

\section{Patients' Distribution Across Study Visits}

Table 2 shows the number of patients with available data at each visit and at the follow-up phone call made in Ramadan.

\section{Administration of Dapagliflozin and Other Concomitant Medications}

A total of 520 patients received dapagliflozin $10 \mathrm{mg}$ for a mean (SD) duration of 10.94 (2.97) months, while one patient received dapagliflozin $5 \mathrm{mg}$ for 14.23 months. Dapagliflozin dose was missing for three patients.

It was reported that 386 patients $(73.7 \%)$ received other antidiabetic medications in addition to dapagliflozin. The most prescribed antidiabetic medication was metformin ( $n=333 ; 63.5 \%)$, followed by DPP-4 inhibitors $(n=224 ; 42.7 \%)$ and sulfonylureas $(n=180$; $34.4 \%)$. A total of 95 patients $(24.6 \%)$ were on antidiabetic monotherapy, 157 (40.7\%) were on dual therapy, $120(31.1 \%)$ were on triple therapy, and $14(3.6 \%)$ were taking more than three antidiabetic medications.

Among 524 patients, 225 (42\%) received antihypertensive medications during the study. The most frequently used classes were angiotensin-converting enzyme inhibitors and angiotensin receptor blockers (ACEIs/ARBs) $(n=170 ; 32.4 \%)$. Table 3 shows a list of all antidiabetic and antihypertensive medications received in concomitance with dapagliflozin.

\section{Safety Outcomes}

Of 527 patients, 65 (12.3\%) experienced 106 adverse events during the observation period. The most commonly reported adverse events were vulvovaginal pruritus $(n=16 ; 3.1 \%)$, dysuria $(n=14 ; 2.7 \%)$, polyuria $(n=7 ; 1.3 \%)$ and UTIs $(n=5 ; 1 \%)$ followed by fatigue $(n=4$; $0.8 \%)$ and hypoglycemia $(n=4 ; 0.8 \%)$. One case of DKA $(0.2 \%)$ was reported during the observation period. This patient was a hypertensive, 59-year-old male with an approximate diabetes duration of 10 years. He was receiving an ARB and a calcium channel blocker (CCB) with no history of DKA prior to receiving dapagliflozin.

The majority of adverse events were non-serious $(n=96 ; 90.5 \%)$. Nine serious adverse events $(8.5 \%)$ were reported; all were recovered. Of those, six were considered by the investigators to be unrelated to dapagliflozin. These included two cases of nephrolithiasis and one case each of pneumonia, gastroenteritis, positional vertigo, and hospitalization for left shoulder arthroscopic rotator cuff tear repair. On the other hand, three serious adverse events were judged by the investigators to be related to dapagliflozin. These were cellulitis in one patient and Fournier's gangrene and testicular abscess in another. Moreover, ten patients were reported to stop dapagliflozin treatment because of experiencing adverse events (Table 4). One patient was missing the seriousness criteria.

Most of the adverse events were of mild severity $(n=79 ; 74.5 \%)$, and 17 events $(16.0 \%)$ were of moderate severity. A total of 57 events $(53.8 \%)$ were considered by the investigator to 
Table 1 Demographic and other baseline characteristics of the study population

\begin{tabular}{|c|c|c|}
\hline Demographics and baseline characteristics & Valid $n=$ & Count (\%) \\
\hline \multicolumn{3}{|l|}{ Sex } \\
\hline Male & 524 & $322(61.5)$ \\
\hline Female & & $202(38.5)$ \\
\hline \multicolumn{3}{|l|}{ Race } \\
\hline Arabs & 524 & $475(90.6)$ \\
\hline Asian & & $37(7.1)$ \\
\hline Of African descent & & $8(1.5)$ \\
\hline Other & & $4(0.8)$ \\
\hline Patients with comorbidity & 521 & $331(63.5)$ \\
\hline Hyperlipidemia & & $266(51.1)$ \\
\hline Hypertension & & $214(41.1)$ \\
\hline Cardiovascular disorders & & $13(2.5)$ \\
\hline Coronary artery disease & & $7(1.4)$ \\
\hline Myocardial infarction & & $3(0.6)$ \\
\hline Stroke & & $2(0.4)$ \\
\hline Heart failure & & $1(0.2)$ \\
\hline UTI (within 12 months) & & $7(1.4)$ \\
\hline Others & & $5(0.96)$ \\
\hline Age, years & 524 & $52.27(14.8)$ \\
\hline Weight, kg & 519 & $87(24)$ \\
\hline Height, $\mathrm{cm}$ & 518 & $167(15)$ \\
\hline BMI, $\mathrm{kg} / \mathrm{m}^{2}$ & 518 & $31.64(7.74)$ \\
\hline T2DM duration, years & 517 & $8.78(6.73)$ \\
\hline
\end{tabular}

Table 2 Patients' distribution across study visits

\begin{tabular}{lllll}
\hline $\begin{array}{l}\text { Baseline } \\
\text { visit }\end{array}$ & $\begin{array}{l}\text { Visit 2 (Month } \\
\mathbf{3} \pm \mathbf{2} \text { weeks) }\end{array}$ & $\begin{array}{l}\text { Visit 3 (Month } \\
\mathbf{6} \pm \mathbf{2} \text { weeks) }\end{array}$ & $\begin{array}{l}\text { Visit 4 (Month } \\
\mathbf{1 2} \pm \mathbf{2} \text { weeks) }\end{array}$ & $\begin{array}{l}\text { Ramadan phone } \\
\text { call } \\
\text { (Unscheduled) }\end{array}$ \\
\hline 527 & 447 & 409 & 367 & 368 \\
\hline
\end{tabular}

be related to dapagliflozin. The vast majority of the reported adverse events $(n=91,85.9 \%)$ were recovered or resolved, while four events $(3.8 \%)$ were recovering, three events $(2.8 \%)$ were not recovered, and one event (0.9\%) recovered with sequala, while the outcomes of seven events $(6.6 \%)$ were unknown. No deaths were reported during the observation period. 
Table 3 Concomitant antidiabetic and antihypertensive medications

\begin{tabular}{lr}
\hline Concomitant antidiabetic and antihypertensive medications $(\boldsymbol{n}=\mathbf{5 2 4})$ & Count (\%) \\
\hline Antidiabetic medications received in addition to dapagliflozin & $333(63.5)$ \\
Biguanides (metformin) & $224(42.7)$ \\
Dipeptidyl-peptidase IV inhibitors & $180(34.4)$ \\
Sulfonylureas & $63(12.0)$ \\
Incretin mimetics/GLP-1 receptor agonists & $18(3.4)$ \\
Thiazolidinediones & $5(1.0)$ \\
a-Glucosidase inhibitors & $2(0.4)$ \\
Meglitinides/prandial glucose regulator/glinides & \\
Antihypertensive medications & $170(32.4)$ \\
ACEI/ARBs ${ }^{\dagger}$ & $60(11.7)$ \\
Calcium channel antagonists & $56(10.7)$ \\
Beta-blockers & $23(4.4)$ \\
Diuretics & $1(0.2)$ \\
Alpha 1 adrenergic receptor antagonists &
\end{tabular}

$G L P-1$ glucagon-like-peptide-1

$\dagger$ ACEI/ARBs: angiotensin-converting enzyme inhibitors and angiotensin receptor blockers

See Tables SD2-SD4 in the electronic supplementary material for details.

No significant difference was found in either age or race between patients with and without adverse events while there was a significantly higher incidence of adverse events among female patients (Table 5).

\section{Efficacy Outcomes}

Mean (SD) HbA1c\% decreased significantly from 8.6 (1.6) \% at baseline to reach 7.4 (1.3) \% $(p<0.0001)$ after 6 months of treatment with dapagliflozin alone or in combination with other antidiabetic medications. When compared to baseline HbA1c, a further decrease in mean (SD) HbA1c\% was observed after 12 months of treatment as it reached 7.2 (1.2) \% $(p<0.0001)$. More details are provided in Table 6.

In addition to HbA1c, the change in the patients' lipid profile at the follow-up visits was assessed compared to the baseline (Table 7).

\section{DISCUSSION}

Despite the availability of a great variety of medications, a significant proportion of people with T2DM are not able to achieve or maintain adequate glycemic control [33]. The recent American Association of Clinical Endocrinology (AACE) 2020 guidelines have recommended SGLT2 inhibitors as the first oral therapy after metformin [21].

Accordingly, adding a medication to control blood glucose levels through an insulin-independent pathway could be advantageous. SGLT2 inhibitors reduce hyperglycemia by blocking the glucose reabsorption in the kidney and therefore promote glucosuria [23]. Dapagliflozin, an SGLT2 inhibitor, is used for T2DM either initially as monotherapy or in combination with other agents in uncontrolled hyperglycemia, without increasing hypoglycemia risk [30, 34, 35].

Previous studies demonstrated the cardiorenal benefits of SGLT2 inhibitors 
Table 4 Patients who stopped dapagliflozin treatment because of experiencing adverse events

\begin{tabular}{llllllll}
\hline Patient & Age & Gender & AE & Seriousness & Severity & Outcome & $\begin{array}{l}\text { Relationship } \\
\text { to dapagliflozin }\end{array}$ \\
\hline 1 & 65 & Female & Abdominal pain & Non-serious & Mild & Unknown & Not related \\
2 & 67 & Female & Glomerular filtration rate decreased & Non-serious & Mild & Unknown & Not related \\
3 & 71 & Male & Polyuria & Non-serious & Moderate & Unknown & Related \\
4 & 70 & Female & Fatigue & Non-serious & Mild & Recovered & Related \\
5 & 48 & Male & Testicular abscess & Serious & Severe & Recovered & Related \\
5 & 48 & Male & Fournier's gangrene & Serious & Severe & Recovered & Related \\
6 & 30 & Male & Urinary tract infection & Non-serious & Moderate & Recovered & Related \\
7 & 63 & Male & Pollakiuria & Non-serious & Mild & Recovered & Not related \\
8 & 57 & Female & Vaginal infection & Non-serious & Mild & Recovered & Not related \\
9 & 56 & Female & Urinary tract infection & Non-serious & Mild & Recovered & Related \\
10 & 59 & Male & Diabetic ketoacidosis & Non-serious & Mild & Recovered & Related \\
\hline
\end{tabular}

(empagliflozin, canagliflozin,

and dapagliflozin), especially in reducing the hospitalization rate due to HF, risk of kidney disease progression, and mortality in patients with T2DM [15-19]. Hence, recent international guidelines for diabetes management have endorsed the use of SGLT2 inhibitors for patients with HF or CKD independent of baseline or target HbA1c [20-22].

The majority of our population were males and Arabs with a median (IQR) age of 52.3 (14.8) years. Two-thirds of the patients were receiving other antidiabetic medications along with dapagliflozin.

Genital infections such as vulvovaginitis are among the common complications of T2DM. Inadequate glycemic control in patients with T2DM results in hyperglycemia and glucosuria, both of which are possible reasons for aggravating vulvovaginitis in this population. Hyperglycemia weakens the body's defense mechanisms, while glucosuria increases the adherence of bacteria to vaginal epithelial cells, thereby providing a suitable environment for microorganisms to thrive. As mentioned earlier, SGLT2 inhibitors normalize the blood glucose levels by increasing urinary glucose excretion, therefore increasing the risk of genital infections. In several meta-analyses and systematic reviews, there was a slight increase in the rate of genitourinary tract infections, including vulvovaginal candida infections and UTIs, when comparing $10 \mathrm{mg}$ dapagliflozin with placebo $[27,36,37]$.

The results of our present study concerning the safety of dapagliflozin showed that out of 524 patients, $65(12.3 \%)$ reported a total of 106 adverse events. These events included vulvovaginal pruritus $(3.1 \%)$, dysuria $(2.7 \%)$, polyuria $(1.3 \%)$, UTI $(1 \%)$, and hypoglycemia $(0.8 \%)$. One case of diabetic ketoacidosis $(0.2 \%)$ was reported. More than $90 \%$ of the adverse events were non-serious. All serious adverse events and the majority of non-serious adverse events were recovered.

Although vulvovaginal pruritus was the most common adverse event in the present study, it is worth mentioning that it was generally not accompanied by an infection. Out of the vulvovaginal pruritus events, three patients presented with vaginal infections ( 2 of these were vulvovaginal candidiasis). In a pooled analysis of safety data derived from 12 randomized, placebo-controlled phase $2 \mathrm{~b} / 3$ trials in inadequately controlled T2DM patients, vulvovaginal pruritus was reported with dapagliflozin $2.5 \mathrm{mg}$, $5 \mathrm{mg}$, and $10 \mathrm{mg}$ at a rate of $1.3 \%, 1.9 \%$, and $1.5 \%$, respectively [38]. The higher rate of 
Table 5 Subgroup comparisons for incidence of adverse events

\begin{tabular}{|c|c|c|c|c|c|}
\hline \multirow[t]{2}{*}{ Parameter $($ valid $n=527)$} & \multicolumn{2}{|c|}{$\begin{array}{l}\text { Patients with adverse events } \\
(n=65)\end{array}$} & \multicolumn{2}{|c|}{$\begin{array}{l}\text { Patients without adverse events } \\
(n=462)\end{array}$} & \multirow[t]{2}{*}{$p$ value } \\
\hline & Count (\%) & $\overline{\text { Median (IQR) }}$ & Count (\%) & Median (IQR) & \\
\hline Age (years) & & $53.99(11.76)$ & & $51.82(15.03)$ & $0.138^{*}$ \\
\hline \multicolumn{6}{|l|}{ Gender } \\
\hline Male & $22(33.8)$ & & $300(64.9)$ & & $<0.001^{* *}$ \\
\hline Female & $43(66.2)$ & & $162(35.1)$ & & \\
\hline \multicolumn{6}{|l|}{ Race } \\
\hline Arab & $62(95.4)$ & & $416(90)$ & & $0.165^{* *}$ \\
\hline Non-Arab & $3(4.6)$ & & $46(10)$ & & \\
\hline
\end{tabular}

${ }^{*}$ Mann-Whitney $U$ test

**Pearson chi-square test

Table 6 Changes in HbA1c\% after 6 and 12 months of treatment with dapagliflozin

\begin{tabular}{llll}
\hline Variables & $\begin{array}{l}\text { Baseline visit } \\
\boldsymbol{n = 5 2 1}\end{array}$ & $\begin{array}{l}\text { After } \mathbf{6} \text { months } \\
\boldsymbol{n}=\mathbf{3 4 5}\end{array}$ & $\begin{array}{l}\text { After 12 months } \\
\boldsymbol{n}=\mathbf{3 2 8}\end{array}$ \\
\hline HbA1c\%, mean (SD) & $8.6(1.6)$ & $7.4(1.3)$ & $7.2(1.2)$ \\
$95 \%$ CI & $(8.5-8.8)$ & $(7.3-7.6)$ & $(7.1-7.4)$ \\
Change from baseline, mean (SD) & - & $-1.09(1.30)^{*}$ & $-1.23(1.39)^{*}$ \\
\hline
\end{tabular}

${ }^{*}$ Wilcoxon signed-rank test revealed $p$ value $<0.0001$

Table 7 Changes in patients' lipid profile after 6 and 12 months of treatment with dapagliflozin

\begin{tabular}{|c|c|c|c|c|c|c|c|}
\hline \multirow[t]{2}{*}{ Variables } & \multicolumn{2}{|c|}{ Baseline visit } & \multicolumn{2}{|c|}{ After 6 months } & \multicolumn{2}{|c|}{ After 12 months } & \multirow[t]{2}{*}{$p$ value } \\
\hline & $\bar{n}$ & Median (IQR) & $\bar{n}$ & Median (IQR) & $n$ & Median (IQR) & \\
\hline $\mathrm{LDL}(\mathrm{mg} / \mathrm{dl})$ & 350 & $100.8(46.4)$ & 153 & $95(50.3)$ & 148 & $88.9(42.5)$ & 0.001 \\
\hline $\mathrm{HDL}(\mathrm{mg} / \mathrm{dl})$ & 292 & $42.3(15.5)$ & 99 & $42.5(11.6)$ & 99 & $46.4(11.6)$ & 0.283 \\
\hline Triglycerides $(\mathrm{mg} / \mathrm{dl})$ & 326 & $150.6(97.4)$ & 131 & $141.7(88.6)$ & 129 & $132.9(79.7)$ & 0.005 \\
\hline Cholesterol (mg/dl) & 306 & $166.3(52.7)$ & 92 & $158.6(38.7)$ & 87 & $150.8(42.5)$ & 0.005 \\
\hline
\end{tabular}

*Wilcoxon signed-rank tests between baseline visit and after 12 months of follow-up

vulvovaginal pruritus reported in our study compared to the pooled analysis might be a result of the higher mean baseline HbA1c level in our population [8.6 (1.6) \% with a maximum value as high as $15.5 \%]$ compared to the pooled population $(8.1-8.4 \%)$.
The rate of dysuria reported in our study $(2.7 \%)$ is moderately higher than those reported in a pooled analysis of patients who received dapagliflozin in 12 placebo-controlled studies (1.6\% and $2.1 \%$ with dapagliflozin $5 \mathrm{mg}$ and $10 \mathrm{mg}$, respectively). On the other hand, the 
incidence of polyuria reported in the present study $(1.3 \%)$ is lower than that reported by the same pooled analysis $(2.9 \%$ and $3.8 \%$ with dapagliflozin $5 \mathrm{mg}$ and $10 \mathrm{mg}$, respectively). Additionally, we reported a rate of $1 \%$ for UTIs, which is much lower than that reported by the same analysis $(5.7 \%$ and $4.3 \%$ with dapagliflozin $5 \mathrm{mg}$ and $10 \mathrm{mg}$, respectively) [39].

In 2015, the FDA issued a warning on the risk of DKA with SGLT2 inhibitors and announced the identification of 73 cases of ketoacidosis in patients with diabetes treated with SGLT2 inhibitors. This FDA announcement has resulted in added warnings to the labels of all SGLT2 inhibitors, including dapagliflozin. Moreover, there was a recent change in the FDA label (prescribing information) for all SGLT2 inhibitors in March 2020. It recommends temporary discontinuation of dapagliflozin at least 3 days before any scheduled surgery to minimize the post-surgical risk of ketoacidosis [40]. Given the seriousness of DKA risk with SGLT2 inhibitors, keeping in mind its incidence in clinical practice is extremely important. In the present study, one patient $(0.2 \%)$ experienced mild DKA. Our results are similar to other previous studies; in a pooled analysis of 21 large placebo-/comparator-controlled clinical trials, one serious adverse event of DKA was reported in the dapagliflozin group (in a patient who received dapagliflozin in addition to metformin and insulin compared to none in the control group). In this analysis, the estimated incidence of DKA was $0.02 \%$ [41].

Dapagliflozin demonstrated favorable glycemic control in T2DM patients for 52 weeks when added to other antidiabetic agents. It was associated with a significant reduction in mean HbA1c from the baseline by - 1.09 (1.39) \% and -1.23 (1.39) \% after 6 and 12 months of treatment, respectively $(p<0.0001)$.

Our results were in accordance with other studies conducted on $10 \mathrm{mg}$ dapagliflozin when used as a dual or triple combination in T2DM. Bailey et al. assessed the efficacy of dapagliflozin combined with metformin for 2 years. They reported a maintained reduction in $\mathrm{HbA1c}$. The mean changes from baseline HbA1c (8.06\%) were $+0.02 \%$ for placebo compared with -
$0.78 \%$ for dapagliflozin at 102 weeks $(p<0.0001)$ [30].

Another meta-analysis assessed dapagliflozin's efficacy at a dose of $10 \mathrm{mg}$ /day. It was associated with a significant reduction in $\mathrm{HbA1c}$ by $-0.54 \% \quad(95 \% \quad \mathrm{CI}-0.67 \%$ to $-0.40 \%$, $p<0.00001)$ after $12-26$ weeks of treatment compared to placebo. After a year, HbA1c reduction was comparable to that at 26 weeks [36]. In another meta-analysis, after 12 to 48 weeks, at a dose of $10-50 \mathrm{mg}$, either as monotherapy or in a combination, the mean reduction in $\mathrm{HbA1c}$ achieved with dapagliflozin ranged from 0.39 to $2.05 \%$ [37].

\section{Study Limitations}

Despite the long duration of follow-up among a varied patient population, the current study could have some limitations as it is a local study performed on a relatively small sample of patients with limited possibilities to assess the occurrence of rare events, including DKA. In addition, the relatively small sample size might have resulted in underestimation of the incidence rate of adverse events. The study was mainly descriptive because of the lack of a comparison group. Considering the observational nature of the study, the patients were treated and observed as a part of the routine practice with no obligations in terms of visit schedules or laboratory investigations. This might have been associated with a relatively large number of drop-out patients at follow-up visits. Accordingly, a significant number of data points related to laboratory investigations were missing at follow-up visits.

\section{CONCLUSIONS}

Results of the current study demonstrate that dapagliflozin is well tolerated, with $1 \%$ of the patients reporting UTIs and $0.2 \%$ reporting DKA (one patient), and effective with significantly improved HbA1c levels after 1 year of treatment. Hence, it can be incorporated into the routine clinical management of T2DM in Saudi Arabia. Future experimental studies with larger samples are required to assess the occurrence of 
rare adverse events that may result from using dapagliflozin.

\section{ACKNOWLEDGEMENTS}

We thank the participants of the study.

Funding. This study was funded by AstraZeneca, which took no part in the analysis or interpretation of the data. AstraZeneca also funded the journal's Rapid Service Fee.

Editorial Assistance. Editorial support for this article was provided by Maha Abulfetoh and Omnia Aboutaleb on behalf of DATACL in CRO and was funded by AstraZeneca.

Authorship. All named authors meet the International Committee of Medical Journal Editors (ICMJE) criteria for authorship for this article, take responsibility for the integrity of the work as a whole, and have given their approval for this version to be published.

Author Contributions. The author, Abdullah M. Alguwaihes, contributed to the study conception and design, in addition to data collection and interpretation. The author also critically reviewed this manuscript for important intellectual content, granted the final approval to be published, and is accountable for all aspects of this work.

List of Investigators. We would like to thank all participating co-investigators for their valuable contribution in this study. Co-investigators in alphabetical order: Abdel Hamid Hassan (International Medical Center), Abdullah Kamal Omer (Dallah Hospital), Ahmed Awad (Dallah Hospital), Ahmed Elsadek (Sulaiman Al Habeeb-Al Rayan), Amin Kemawy (King Abdulaziz University Medical Center), Amjad Kafelghazal (Sulaiman Al Habeeb-Olaya), Heba Bzeih (International Medical center), Hiba Adam (King Khalid University Hospital), Hilali Hassan Hilali (Dallah Hospital), Maha Hemadn (Sulaiman Al Habeeb-Al Rayan), Mohammed Batais (King Khalid University Hospital), Mowaia Mustafa (Sulaiman Al Habeeb-Olaya), Naweed
Alzamman (Tayba University Hospital), Nidal Abu Diab (Specialized Medical Center Hospital), Ola Jarrad (Specialized Medical Center Hospital), Osman Wardy (Sulaiman Al Habeeb-Al Rayan), Rafif Farahat (Sulaiman Al Habeeb-Al Takhsusi), Saeed Taha (Sulaiman Al Habeeb-Al Takhsusi), Saher Safarini (Dallah Hospital), Said Khader (Sulaiman Al Habeeb-Olaya Medical Complex), Salah Kouta (Sulaiman Al Habeeb-Al Sewedy), Samia El Shiekh (Dallah Hospital), Samir Arif (King Khalid University Hospital), Syed Salman (Specialized Medical Center Hospital), Tarek Motawaea (Specialized Medical Center Hospital), Turky Almugbil (King Khalid University Hospital), and Waleed Maghawry Saleh (King Abdulaziz University Medical Center).

Prior Presentation. Baseline characteristics were published in 2020; DOI: 10.4103/ JNSM.JNSM_54_19.

Disclosures. The author, Abdullah M. Alguwaihes, has received speaker honoraria and was part of advisory boards of the following pharmaceutical companies: AstraZeneca, Boehringer Ingelheim, Eli Lilly, Novo Nordisk, and Sanofi. In addition, research support from AstraZeneca and Novo Nordisk.

Compliance with Ethics Guidelines. This study was approved by the appropriate institutional ethics committees of all study sites. Namely: Dallah Hospital (reference number: 17/0979/IRB), International Medical Center (reference number: 2017-11-081), King Abdulaziz University Medical Center (reference number: 159-18), King Khalid University Hospital (reference number: 17/0253/IRB), Specialized Medical Center Hospital (reference number: SMCIRB0004-2017), Sulaiman Al Habeeb Hospital (reference number: 17/0498/ IRB), and Tayba University Hospital (reference number: 17/0253/IRB). See Table SD1 in the electronic supplementary material for details. The study was conducted in accordance with the ethical principles of the Declaration of Helsinki and Good Pharmacoepidemiology Practice. The study also adhered to the laws, regulations and any relevant guidelines of Saudi 
Arabia. Before conducting any research-related activities, all patients provided personally signed and dated informed consent documents, indicating that the patient or their legally acceptable representative was informed of all pertinent aspects of the study and the patients consented to participate in this study. Consent for publication: all patients consented that their data may be shared after their anonymization for the purposes of scientific and medical research (e.g., with researchers, to allow public access to study information, for sharing results with patients who participate in study or in publications).

Data Availability. The datasets used during the current study are available from the corresponding author upon request.

Open Access. This article is licensed under a Creative Commons Attribution-NonCommercial 4.0 International License, which permits any non-commercial use, sharing, adaptation, distribution and reproduction in any medium or format, as long as you give appropriate credit to the original author(s) and the source, provide a link to the Creative Commons licence, and indicate if changes were made. The images or other third party material in this article are included in the article's Creative Commons licence, unless indicated otherwise in a credit line to the material. If material is not included in the article's Creative Commons licence and your intended use is not permitted by statutory regulation or exceeds the permitted use, you will need to obtain permission directly from the copyright holder. To view a copy of this licence, visit http://creativecommons.org/licenses/by$\mathrm{nc} / 4.0 /$.

\section{REFERENCES}

1. Ogurtsova K, da Rocha Fernandes JD, Huang Y, et al. IDF diabetes atlas: global estimates for the prevalence of diabetes for 2015 and 2040. Diabetes Res Clin Pract. 2017;128:40-50.
2. International Diabetes Federation. IDF Diabetes Atlas. 9th ed. Brussels: International Diabetes Federation; 2019.

3. Boyle JP, Engelgau MM, Thompson TJ, et al. Estimating prevalence of type 1 and type 2 diabetes in a population of African Americans with diabetes mellitus. Am J Epidemiol. 1999;149:55-63.

4. Bruno G, Runzo C, Cavallo-Perin P, et al. Incidence of type 1 and type 2 diabetes in adults aged 30-49 years. Diabetes Care. 2005;28:2613-9.

5. Evans JM, Newton RW, Ruta DA, MacDonald TM, Morris AD. Socio-economic status, obesity and prevalence of type 1 and type 2 diabetes mellitus. Diabet Med. 2000;17:478-80.

6. World Health Organization. Global status report on noncommunicable diseases 2014: attaining the nine global noncommunicable diseases targets; a shared responsibility. Geneva: World Health Organization; 2014.

7. Roger VL, Go AS, Lloyd-Jones DM, et al. Heart disease and stroke statistics-2011 update: a report from the American Heart Association. Circulation. 2011;123:e18-209.

8. Donahoe SM, Stewart GC, McCabe CH, et al. Diabetes and mortality following acute coronary syndromes. JAMA. 2007;298:765-75.

9. Ahmad FS, Ning H, Rich JD, Yancy CW, Lloyd-Jones DM, Wilkins JT. Hypertension, obesity, diabetes, and heart failure-free survival: the cardiovascular disease lifetime risk pooling project. JACC Heart Fail. 2016;4:911-9.

10. Ritz E, Orth SR. Nephropathy in patients with type 2 diabetes mellitus. N Engl J Med. 1999;341: 1127-33.

11. Wing RR, Bolin P, Brancati FL, et al. Cardiovascular effects of intensive lifestyle intervention in type 2 diabetes. N Engl J Med. 2013;369:145-54.

12. Lean MJ, Leslie WS, Barnes AC, et al. Primary careled weight management for remission of type 2 diabetes (DiRECT): an open-label, cluster-randomised trial. Lancet. 2018;391:541-51.

13. Umpierre D, Ribeiro PA, Kramer CK, et al. Physical activity advice only or structured exercise training and association with HbA1c levels in type 2 diabetes: a systematic review and meta-analysis. JAMA. 2011;305:1790-9.

14. Rubino F, Nathan DM, Eckel RH, et al. Metabolic surgery in the treatment algorithm for type 2 diabetes: a joint statement by International Diabetes Organizations. Diabetes Care. 2016;39:861-77. 
15. Neal B, Perkovic V, Mahaffey KW, et al. Canagliflozin and cardiovascular and renal events in type 2 diabetes. N Engl J Med. 2017;377:644-57.

16. Zinman B, Wanner C, Lachin JM, et al. Empagliflozin, cardiovascular outcomes, and mortality in type 2 diabetes. N Engl J Med. 2015;373:2117-28.

17. Wiviott SD, Raz I, Bonaca MP, et al. Dapagliflozin and cardiovascular outcomes in type 2 diabetes. N Engl J Med. 2019;380:347-57.

18. Perkovic V, Jardine MJ, Neal B, et al. Canagliflozin and renal outcomes in type 2 diabetes and nephropathy. N Engl J Med. 2019;380:2295-306.

19. Wanner C, Inzucchi SE, Lachin JM, et al. Empagliflozin and progression of kidney disease in type 2 diabetes. N Engl J Med. 2016;375:323-34.

20. American Diabetes Association. 1. Improving care and promoting health in populations: standards of medical care in diabetes-2020. Diabetes Care. 2020;43(1):S7-13.

21. Garber AJ, Handelsman Y, Grunberger G, et al. Consensus statement by the American Association of Clinical Endocrinologists and America College of Endocrinology on the comprehensive type 2 diabetes management algorithm-2020 executive summary. Endocr Pract. 2020;26:107-39.

22. Cosentino F, Grant PJ, Aboyans V, et al. 2019 ESC guidelines on diabetes, pre-diabetes, and cardiovascular diseases developed in collaboration with the EASD. Eur Heart J. 2020;41:255-323.

23. DeSantis Anthony. Sodium-glucose co-transporter 2 inhibitors for the treatment of type 2 diabetes mellitus. In: Nathan DM, Mulder JE, eds. UpToDate. Waltham (MA): UpToDate; 2020. http://www. uptodate.com. Accessed 24 Oct 2020.

24. European Medicines Agency. Forxiga (dapagliflozin) An overview of Forxiga and why it is authorised in the EU. 2021. https://www.ema. europa.eu/en/documents/overview/forxiga-eparmedicine-overview_en.pdf. Accessed 25 Oct 2020.

25. McMurray JJV, Solomon SD, Inzucchi SE, et al. Dapagliflozin in patients with heart failure and reduced ejection fraction. N Engl J Med. 2019;381: 1995-2008.

26. Heerspink HJL, Stefánsson BV, Correa-Rotter R, et al. Dapagliflozin in patients with chronic kidney Disease. N Engl J Med. 2020;383:1436-46.

27. Clar C, Gill JA, Court R, et al. Systematic review of SGLT2 receptor inhibitors in dual or triple therapy in type 2 diabetes. BMJ Open. 2012;2:e001007.
28. Douros A, Lix LM, Fralick M, et al. Sodium-glucose cotransporter- 2 inhibitors and the risk for diabetic ketoacidosis: a multicenter cohort study. Ann Intern Med. 2020;173:417-25.

29. Taylor SI, Blau JE, Rother KI. SGLT2 inhibitors may predispose to ketoacidosis. J Clin Endocrinol Metab. 2015;100:2849-52.

30. Bailey CJ, Gross JL, Hennicken D, Iqbal N, Mansfield TA, List JF. Dapagliflozin add-on to metformin in type 2 diabetes inadequately controlled with metformin: a randomized, double-blind, placebocontrolled 102-week trial. BMC Med. 2013;11:43.

31. Nyirjesy P, Zhao Y, Ways K, Usiskin K. Evaluation of vulvovaginal symptoms and Candida colonization in women with type 2 diabetes mellitus treated with canagliflozin, a sodium glucose co-transporter 2 inhibitor. Curr Med Res Opin. 2012;28:1173-8.

32. Alguwaihes AM. Baseline characteristics of patients with type 2 diabetes mellitus initiating Dapagliflozin in Saudi Arabia: results from postauthorization safety study. J Nat Sci Med. 2020;3:210-3.

33. Seufert J. SGLT2 inhibitors-an insulin-independent therapeutic approach for treatment of type 2 diabetes: focus on canagliflozin. Diabetes Metab Syndr Obes. 2015;8:543-54.

34. Ferrannini E, Ramos SJ, Salsali A, Tang W, List JF. Dapagliflozin monotherapy in type 2 diabetic patients with inadequate glycemic control by diet and exercise: a randomized, double-blind, placebocontrolled, phase 3 trial. Diabetes Care. 2010;33: 2217-24.

35. Strojek K, Yoon KH, Hruba V, Elze M, Langkilde AM, Parikh S. Effect of Dapagliflozin in patients with type 2 diabetes who have inadequate glycaemic control with glimepiride: a randomised, 24-week, double-blind, placebo-controlled trial. Diabetes Obes Metab. 2011;13:928-38.

36. Musso G, Gambino R, Cassader M, Pagano G. A novel approach to control hyperglycemia in type 2 diabetes: sodium glucose co-transport (SGLT) inhibitors: systematic review and meta-analysis of randomized trials. Ann Med. 2012;44:375-93.

37. Zhang $\mathrm{M}$, Zhang $\mathrm{L}, \mathrm{Wu} \mathrm{B}$, Song $\mathrm{H}$, An Z, Li S. Dapagliflozin treatment for type 2 diabetes: a systematic review and meta-analysis of randomized controlled trials. Diabetes Metab Res Rev. 2014;30: 204-21.

38. Johnsson KM, Ptaszynska A, Schmitz B, Sugg J, Parikh SJ, List JF. Urinary tract infections in patients with diabetes treated with Dapagliflozin. J Diabetes Complicat. 2013;27:473-8. 
39. Ptaszynska A, Johnsson KM, Parikh SJ, de Bruin TW, Apanovitch AM, List JF. Safety profile of Dapagliflozin for type 2 diabetes: pooled analysis of clinical studies for overall safety and rare events. Drug Saf. 2014;37:815-29.

40. U.S. Food and Drug Administration. FDA revises labels of SGLT2 inhibitors for diabetes to include warnings about too much acid in the blood and serious urinary tract infections. 2015. https://www.
fda.gov/files/drugs/published/FDA-revises-labels-ofSGLT2-inhibitors-for-diabetes-to-include-warningsabout-too-much-acid-in-the-blood-and-seriousurinary-tract-infections.pdf. Accessed 24 Oct 2020.

41. Jabbour S, Seufert J, Scheen A, Bailey CJ, Karup C, Langkilde AM. Dapagliflozin in patients with type 2 diabetes mellitus: a pooled analysis of safety data from phase IIb/III clinical trials. Diabetes Obes Metab. 2018;20:620-8. 